Homology, Homotopy and Applications, vol.13(2), 2011, pp.293-300

\title{
ON THE ORIENTABILITY OF THE SLICE FILTRATION
}

\author{
PABLO PELAEZ
}

(communicated by Daniel Dugger)

\begin{abstract}
Let $X$ be a Noetherian separated scheme of finite Krull dimension. We show that the layers of the slice filtration in the motivic stable homotopy category $\mathcal{S H}$ are strict modules over Voevodsky's algebraic cobordism spectrum. We also show that the zero slice of any commutative ring spectrum in $\mathcal{S H}$ is an oriented ring spectrum in the sense of Morel, and that its associated formal group law is additive. As a consequence, we deduce that with rational coefficients the slices are in fact motives in the sense of Cisinski-Déglise [1] and have transfers if the base scheme is excellent. This proves a conjecture of Voevodsky $[\mathbf{2 5}$, Conjecture 11].
\end{abstract}

\section{Introduction}

Let $X$ be a Noetherian separated scheme of finite Krull dimension, and $\mathcal{M}_{X}$ be the category of pointed simplicial presheaves in the smooth Nisnevich site $S m_{X}$ over $X$ equipped with the Quillen model structure [16] introduced by Morel-Voevodsky [7]. We define $T$ in $\mathcal{M}_{X}$ as the pointed simplicial presheaf represented by $S^{1} \wedge \mathbb{G}_{m}$, where $\mathbb{G}_{m}$ is the multiplicative group $\mathbb{A}_{X}^{1}-\{0\}$ pointed by 1 , and $S^{1}$ denotes the simplicial circle. Let $\operatorname{Spt}\left(\mathcal{M}_{X}\right)$ denote the category of symmetric $T$-spectra on $\mathcal{M}_{X}$ equipped with Jardine's motivic model structure [4]. The homotopy category of $\operatorname{Spt}\left(\mathcal{M}_{X}\right)$ is a triangulated category which will be denoted by $\mathcal{S H}$.

Given an integer $q \in \mathbb{Z}$, we consider the following family of symmetric $T$-spectra:

$$
C_{\text {eff }}^{q}=\left\{F_{n}\left(S^{r} \wedge \mathbb{G}_{m}^{s} \wedge U_{+}\right) \mid n, r, s \geqslant 0 ; s-n \geqslant q ; U \in S m_{X}\right\},
$$

where $F_{n}$ is the left adjoint to the $n$-evaluation functor

$$
e v_{n}: \operatorname{Spt}\left(\mathcal{M}_{X}\right) \rightarrow \mathcal{M}_{X} .
$$

Voevodsky [25] defines the slice filtration as the following family of triangulated subcategories of $\mathcal{S H}$ :

$$
\cdots \subseteq \Sigma_{T}^{q+1} \mathcal{S} \mathcal{H}^{e f f} \subseteq \Sigma_{T}^{q} \mathcal{S} \mathcal{H}^{e f f} \subseteq \Sigma_{T}^{q-1} \mathcal{S H} \mathcal{H}^{e f f} \subseteq \cdots,
$$

where $\Sigma_{T}^{q} \mathcal{S} \mathcal{H}^{\text {eff }}$ is the smallest full triangulated subcategory of $\mathcal{S H}$ which contains $C_{\text {eff }}^{q}$ and is closed under arbitrary coproducts.

Received March 8, 2010, revised November 1, 2011; published on December 6, 2011. 2000 Mathematics Subject Classification: 14F42, 55N22.

Key words and phrases: algebraic cobordism, $K$-theory, mixed motive, oriented cohomology theory, rigid homotopy group, slice filtration, transfer.

Article available at http://intlpress.com/HHA/v13/n2/a17 and doi:10.4310/HHA.2011.v13.n2.a17 Copyright (C) 2011, International Press. Permission to copy for private use granted. 
It follows from the work of Neeman $[\mathbf{8}, \mathbf{9}]$ that the inclusion

$$
i_{q}: \Sigma_{T}^{q} \mathcal{S} \mathcal{H}^{e f f} \rightarrow \mathcal{S H}
$$

has a right adjoint $r_{q}: \mathcal{S H} \rightarrow \Sigma_{T}^{q} \mathcal{S H}{ }^{e f f}$, and that the following functors

$$
\begin{aligned}
& f_{q}: \mathcal{S H} \rightarrow \mathcal{S H} \\
& s_{q}: \mathcal{S H} \rightarrow \mathcal{S H}
\end{aligned}
$$

are triangulated, where $f_{q}$ is defined as the composition $i_{q} \circ r_{q}$, and $s_{q}$ is characterized by the fact that for every $E \in \operatorname{Spt}\left(\mathcal{M}_{X}\right)$, we have the following distinguished triangle in $\mathcal{S H}$ :

$$
f_{q+1} E \stackrel{\rho_{q}^{E}}{\longrightarrow} f_{q} E \stackrel{\pi_{q}^{E}}{\longrightarrow} s_{q} E \longrightarrow \Sigma_{T}^{1,0} f_{q+1} E .
$$

We will refer to $f_{q} E$ as the $(q-1)$-connective cover of $E$, and to $s_{q} E$ as the $q$-slice of $E$. It follows directly from the construction that the $q$-slice of $E$ is right orthogonal with respect to $\Sigma_{T}^{q+1} \mathcal{S} \mathcal{H}^{e f f}$, i.e.,

$$
\operatorname{Hom}_{\mathcal{S H}}\left(K, s_{q} E\right)=0
$$

for every $K$ in $\Sigma_{T}^{q+1} \mathcal{S} \mathcal{H}^{e f f}$.

The results of Section 4 depend on material from [1], which to date has only appeared in preprint form.

\section{Acknowledgements}

The author would like to warmly thank Frédéric Déglise for several useful conversations and suggestions, as well as for putting in his hands the technical tools from [1] and [2], and also thank Denis-Charles Cisinski for bringing to his attention the argument which allowed him to extend Theorem 4.3 from geometrically unibranch base schemes to arbitrary excellent schemes.

\section{Strict $M G L$-modules}

In this section we will show that all the slices have a canonical structure of strict modules in $\operatorname{Spt}\left(\mathcal{M}_{X}\right)$ over Voevodsky's algebraic cobordism spectrum.

Let $A$ be a cofibrant ring spectrum with unit in $\operatorname{Spt}\left(\mathcal{M}_{X}\right)$, and $A$-mod be the category of left $A$-modules in $\operatorname{Spt}\left(\mathcal{M}_{X}\right)$. The work of Jardine [4, Proposition 4.19] and Hovey [3, Corollary 2.2] implies that the adjunction

$$
(A \wedge-, U, \varphi): \operatorname{Spt}\left(\mathcal{M}_{X}\right) \rightarrow A-\bmod
$$

induces a Quillen model structure $S p t^{A}\left(\mathcal{M}_{X}\right)$ in $A$-mod; this means that a map $f: M \rightarrow N$ in $S p t^{A}\left(\mathcal{M}_{X}\right)$ is a weak equivalence or a fibration if and only if $U f$ is a weak equivalence or a fibration in $\operatorname{Spt}\left(\mathcal{M}_{X}\right)$.

It is easy to see that the homotopy category $\mathcal{S H}^{A}$ of $S p t^{A}\left(\mathcal{M}_{X}\right)$ is a triangulated category [13, Proposition 3.5.3].

Definition 2.1. Let $E$ be a spectrum in $\mathcal{S H}$. We say that $E$ is effective if $E$ belongs to the triangulated category $\Sigma_{T}^{0} \mathcal{S H}{ }^{\text {eff }}$ defined above. 
Theorem 2.2. Let $A$ be an effective cofibrant ring spectrum with unit $u^{A}: \mathbf{1} \rightarrow A$ in $\operatorname{Spt}\left(\mathcal{M}_{X}\right)$. If $s_{0}\left(u^{A}\right)$ is an isomorphism in $\mathcal{S H}$, then for every $q \in \mathbb{Z}$ the functor

$$
s_{q}: \mathcal{S H} \rightarrow \mathcal{S H}
$$

factors (up to a canonical isomorphism) through $\mathcal{S H}^{A}$

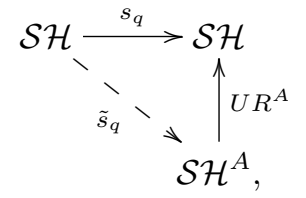

where $R^{A}$ denotes a fibrant replacement functor in $\operatorname{Spt}^{A}\left(\mathcal{M}_{X}\right)$.

Proof. This follows directly from [13, Theorem 3.6.20 and Lemma 3.6.21] or [11, Theorem 2.1(vi)].

The following proposition was proved by Voevodsky $[\mathbf{2 5}$, p. 10, $\S 3.4]$ and Spitzweck [19, Corollaries 3.2 and 3.3]:

Proposition 2.3 (Voevodsky). Let MGL denote Voevodsky's algebraic cobordism spectrum [24]. Then $M G L$ is effective and its unit map $u^{M G L}: \mathbf{1} \rightarrow M G L$ induces an isomorphism on the zero slices in $\mathcal{S H}$

$$
s_{0}\left(u^{M G L}\right): s_{0} \mathbf{1} \cong s_{0} M G L .
$$

Now we can state the main result of this section.

Theorem 2.4. Let $q \in \mathbb{Z}$ denote an arbitrary integer and $E$ denote an arbitrary symmetric T-spectrum in $\operatorname{Spt}\left(\mathcal{M}_{X}\right)$. Then the q-slice $s_{q} E$ of $E$ is equipped with a canonical structure of $M G L$-module in $\operatorname{Spt}\left(\mathcal{M}_{X}\right)$. This implies that over any base scheme, the slices are always oriented cohomology theories in the sense of Déglise [2, Example 2.12(2)].

Proof. This follows immediately from Theorem 2.2 and Proposition 2.3.

Remark 2.5. One of the interesting consequences of Theorem 2.4 is the fact that over any Noetherian separated base scheme of finite Krull dimension, once we pass to the slices it is possible to apply all the formalism developed by Déglise in [2], e.g., Chern classes and the Gysin triangle.

\section{Oriented ring spectra and formal group laws}

In this section we will show that given a commutative ring spectrum $E$ in $\mathcal{S H}$, its zero slice $s_{0} E$ is an oriented ring spectrum (in the sense of Morel [21, Definition 3.1]) with additive formal group law in $\mathcal{S H}$. To simplify the notation we will denote by $\mathbb{P}^{n}$ the trivial projective bundle of rank $n$ over our base scheme $X$. 
Definition 3.1. Let $E$ be a commutative ring spectrum in $\mathcal{S H}$ with unit

$$
u^{E}: \mathbf{1} \rightarrow E .
$$

We say that $E$ is an oriented ring spectrum if there exists an element $x_{E}$ in

$$
\operatorname{Hom}_{\mathcal{S H}}\left(F_{0}\left(\mathbb{P}^{\infty}\right), S^{1} \wedge \mathbb{G}_{m} \wedge E\right),
$$

where $\mathbb{P}^{\infty}$ is the colimit of the diagram

$$
\mathbb{P}^{1} \rightarrow \mathbb{P}^{2} \rightarrow \cdots \rightarrow \mathbb{P}^{n} \rightarrow \cdots
$$

given by the inclusions of the respective hyperplanes at infinity, such that $x_{E}$ pulls back to the following composition:

$$
F_{0}\left(\mathbb{P}^{1}\right) \cong F_{0}\left(S^{1} \wedge \mathbb{G}_{m}\right) \stackrel{i d \wedge u^{E}}{\longrightarrow} F_{0}\left(S^{1} \wedge \mathbb{G}_{m}\right) \wedge E \cong S^{1} \wedge \mathbb{G}_{m} \wedge E .
$$

The following proposition is classical:

Proposition 3.2 (cf. $[\mathbf{1 4}, \mathbf{1 5}, \mathbf{2 1}])$. Let $\left(E, x_{E}\right)$ be an oriented ring spectrum in $\mathcal{S H}$, and let $m: \mathbb{P}^{\infty} \times \mathbb{P}^{\infty} \rightarrow \mathbb{P}^{\infty}$ be the map induced by the corresponding Segre embeddings. The pullback of $x_{E}$ along $m$, is a formal group law $F_{E}$

$$
F_{E}=\sum_{i+j \geqslant 1} c_{i j} x^{i} y^{j}
$$

where the coefficients $c_{i j}$ are elements in the abelian group

$$
\operatorname{Hom}_{\mathcal{S H}}\left(F_{0}\left(S^{i+j-1} \wedge \mathbb{G}_{m}^{i+j-1}\right), E\right),
$$

and $x$ (resp. $y$ ) is the pullback of $x_{E}$ along the projection in the first factor $p_{1}: \mathbb{P}^{\infty} \times$ $\mathbb{P}^{\infty} \rightarrow \mathbb{P}^{\infty}$ (resp. second factor).

Lemma 3.3. Let $E$ be a commutative ring spectrum in $\mathcal{S H}$ with unit $u^{E}: \mathbf{1} \rightarrow E$. Then its zero slice $s_{0} E$ is also a commutative ring spectrum in $\mathcal{S H}$, and the induced map

$$
s_{0}\left(u^{E}\right): s_{0} \mathbf{1} \rightarrow s_{0} E
$$

is a map of ring spectra in $\mathcal{S H}$.

Proof. The fact that $s_{0} E$ is a ring spectrum in $\mathcal{S H}$ follows from [13, Theorem 3.6.13]. On the other hand, the naturality of the pairings constructed in [13, Theorem 3.6.9] implies that $s_{0} E$ is also commutative in $\mathcal{S H}$ and that $s_{0}\left(u^{E}\right)$ is a map of ring spectra in $\mathcal{S H}$.

Lemma 3.4. The natural map

$$
\pi_{0}^{M G L}: M G L \cong f_{0} M G L \rightarrow s_{0} M G L
$$

is a map of ring spectra in $\mathcal{S H}$.

Proof. By Proposition 2.3 we deduce that $M G L$ is naturally isomorphic to $f_{0} M G L$ in $\mathcal{S H}$. On the other hand, Theorem 3.6.10(3) in [13] implies that $\pi_{0}^{M G L}$ is a map of ring spectra in $\mathcal{S H}$. 
Theorem 3.5. Let $E$ be a commutative ring spectrum in $\mathcal{S H}$. Then its zero slice $s_{0} E$ is an oriented ring spectrum in the sense of Morel and it is equipped with a canonical orientation given by the following composition:

$$
M G L \stackrel{\pi_{0}^{M G L}}{\longrightarrow} s_{0} M G L \stackrel{\left(s_{0}\left(u^{M G L}\right)\right)^{-1}}{\longrightarrow} s_{0} \mathbf{1} \stackrel{s_{0}\left(u^{E}\right)}{\longrightarrow} s_{0} E .
$$

Furthermore, the associated formal group law $F_{s_{0} E}$ of $s_{0} E$ is additive.

Proof. The universality of $M G L$ (cf. [21, Theorem 4.3], [10, Theorem 2.7] and [20, Proposition A.2]) implies that in order to show that the map defined above gives an orientation for $s_{0} E$, it is enough to see that all the maps are in fact maps of ring spectra in $\mathcal{S H}$. But this follows directly from Proposition 2.3 together with Lemmas 3.3 and 3.4 .

On the other hand, the formal group law of $s_{0} E$

$$
F_{s_{0} E}=\sum_{i+j \geqslant 1} c_{i j} x^{i} y^{j}
$$

has coefficients $c_{i j}$ which by construction are in the abelian group

$$
\operatorname{Hom}_{\mathcal{S H}}\left(F_{0}\left(S^{i+j-1} \wedge \mathbb{G}_{m}^{i+j-1}\right), s_{0} E\right) .
$$

However, if $i+j>1$ then $F_{0}\left(S^{i+j-1} \wedge \mathbb{G}_{m}^{i+j-1}\right)$ is automatically in $\Sigma_{T}^{1} \mathcal{S} \mathcal{H}^{e f f}$; hence

$$
\operatorname{Hom}_{\mathcal{S H}}\left(F_{0}\left(S^{i+j-1} \wedge \mathbb{G}_{m}^{i+j-1}\right), s_{0} E\right)=0
$$

since $s_{0} E$ is right orthogonal with respect to $\Sigma_{T}^{1} \mathcal{S} \mathcal{H}^{\text {eff }}$.

Therefore, the formal group law of $s_{0} E$

$$
F_{s_{0} E}=\sum_{i+j \geqslant 1} c_{i j} x^{i} y^{j}=x+y
$$

is additive, as we wanted.

\section{Applications}

In this section we will show that with rational coefficients all the slices $s_{q}(E) \otimes \mathbf{1}_{\mathbb{Q}}$ are in a natural way motives in the sense of Cisinski-Déglise [1]. As a consequence we will deduce that over an excellent base scheme the presheaves of rational rigid homotopy groups have transfers; this proves a conjecture of Voevodsky [25, Conjecture 11].

\subsection{Slices and the Cisinski-Déglise category of motives}

Let

$$
\mathbf{H}_{\mathbb{B}, X}=K G L_{X}^{(0)} \in \operatorname{Spt}\left(\mathcal{M}_{X}\right)
$$

denote the Beilinson motivic cohomology spectrum constructed by Riou in [17]. The work of Cisinski-Déglise shows, in particular, that $\mathbf{H}_{\mathbb{B}, X}$ is a commutative cofibrant ring spectrum in $\operatorname{Spt}\left(\mathcal{M}_{X}\right)$ (cf. [1, Corollary 13.2.6]); and that the homotopy category of $\mathbf{H}_{\mathbb{B}, X}$-modules $\mathcal{S} \mathcal{H}^{\mathbf{H}_{\mathbb{B}, X}}$ is naturally equivalent to the Cisinski-Déglise category of motives $D M_{\mathbb{B}, X}$ (cf. [1, Theorem 13.2.9]). 
Theorem 4.1. If we consider rational coefficients, then the zero slice of the sphere spectrum $s_{0}(\mathbf{1}) \otimes \mathbf{1}_{\mathbb{Q}}$ is equipped with a unique structure of $\mathbf{H}_{\mathbb{B}, X}$-algebra in $\operatorname{Spt}\left(\mathcal{M}_{X}\right)$.

In particular, there exists a unique map $\eta_{s_{0}(\mathbf{1}) \otimes \mathbf{1}_{\mathbb{Q}}}$ of ring spectra in $\mathcal{S H}$ such that the following diagram is commutative:

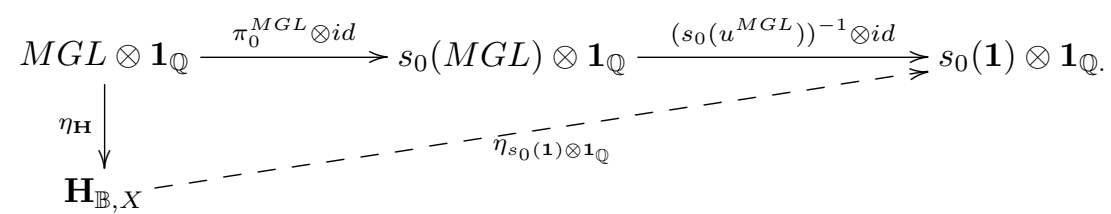

Proof. By Theorem 3.5 we deduce that $s_{0}(\mathbf{1})$ is orientable. Therefore, the result follows directly from Corollary 13.2.15(Ri),(Rii),(Riii) in [1] .

Theorem 4.2. Let $q \in \mathbb{Z}$ denote an arbitrary integer and $E$ denote an arbitrary symmetric T-spectrum in $\operatorname{Spt}\left(\mathcal{M}_{X}\right)$. Then the q-slice of $E$ with rational coefficients $s_{q}(E) \otimes \mathbf{1}_{\mathbb{Q}}$, is equipped with a canonical structure of $\mathbf{H}_{\mathbb{B}, X}$-module in $\operatorname{Spt}\left(\mathcal{M}_{X}\right)$. This implies that over any base scheme, the slices with rational coefficients $s_{q}(E) \otimes \mathbf{1}_{\mathbb{Q}}$ are motives in the sense of Cisinski-Déglise.

Proof. Using Corollary 13.2.15(i),(iv),(v) in [1] , we observe that it is enough to show that $s_{q}(E) \otimes \mathbf{1}_{\mathbb{Q}}$ is a $\mathbf{H}_{\mathbb{B}, X}$-module in $\mathcal{S H}$. On the other hand, by Theorem 4.1 we just need to check that $s_{q}(E) \otimes \mathbf{1}_{\mathbb{Q}}$ is a $s_{0}(\mathbf{1}) \otimes \mathbf{1}_{\mathbb{Q}}$-module in $\mathcal{S H}$. Finally, this follows directly from Theorem 3.6.14(6) in [13].

\subsection{Rational rigid homotopy groups}

Given a symmetric $T$-spectrum $E$ in $\operatorname{Spt}\left(\mathcal{M}_{X}\right)$, Voevodsky defines the presheaves of rigid homotopy groups $\pi_{p, q}^{r i g}(E)$ on $S m_{X}$ as follows:

$$
\begin{aligned}
\pi_{p, q}^{r i g}(E): S m_{X} & \longrightarrow \text { Abelian Groups } \\
U & \longmapsto \operatorname{Hom}_{\mathcal{S H}}\left(F_{0}\left(S^{p} \wedge \mathbb{G}_{m}^{q} \wedge U_{+}\right), s_{q} E\right) .
\end{aligned}
$$

Conjecture 11 in [25] claims that these presheaves have transfers.

Theorem 4.3. Let $p, q \in \mathbb{Z}$ denote arbitrary integers and $E$ denote an arbitrary symmetric T-spectrum in $\operatorname{Spt}\left(\mathcal{M}_{X}\right)$. Furthermore, assume that the base scheme $X$ is excellent. Then the presheaves of rigid homotopy groups of $E$ with rational coefficients $\pi_{p, q}^{r i g}(E) \otimes \mathbb{Q}$ have transfers.

Proof. Clearly, it suffices to show that $s_{q}(E) \otimes \mathbf{1}_{\mathbb{Q}}$ has transfers. Now, Theorem 4.2 implies that $s_{q}(E) \otimes \mathbf{1}_{\mathbb{Q}}$ is in $D M_{\mathbb{B}, X}$. Since we are assuming that $X$ is excellent, Theorem 15.1.2 in [1] implies that $D M_{\mathbb{B}, X}$ is naturally equivalent to the category of motives $D M_{\mathrm{qfh}, X}$ constructed using $q f h$-sheaves with rational coefficients. Thus, the result follows from Theorem 3.3.8 in [22] (see also Proposition 9.5.5 in [1]), which implies than every $q f h$-sheaf is canonically equipped with transfers.

Remark 4.4. Theorem 4.3 was proved using the functoriality of the slice filtration in [12, Theorem 4.4] for schemes defined over a field of characteristic zero; on the other hand, if the base scheme $X$ is smooth over a perfect field $k$, then Theorem 4.3 
holds even with integral coefficients (cf. [13, Theorem 3.6.22]). Both proofs rely on the computation of Levine [5] and Voevodsky [23] for the zero slice of the sphere spectrum, as well as on the work of Röndigs-Østvær [18].

The analogue of this question for the category of $S^{1}$-spectra is studied by Levine in $[6]$.

\section{References}

[1] D.-C. Cisinski and F. Déglise, Triangulated categories of mixed motives, preprint (2009), arXiv:0912.2110.

[2] F. Déglise, Around the Gysin triangle. II, Doc. Math. 13 (2008), 613-675. MR 2466188 (2009m:14025)

[3] M. Hovey, Monoidal model categories, preprint (1998), arXiv:math.AT/ 9803002.

[4] J. F. Jardine, Motivic symmetric spectra, Doc. Math. 5 (2000), 445-553 (electronic). MR 1787949 (2002b:55014)

[5] M. Levine, The homotopy coniveau tower, J. Topol. 1 (2008), no. 1, 217-267. MR 2365658 (2008j:14013)

[6] M. Levine, Slices and transfers, Doc. Math. 2010, Extra volume: Andrei A. Suslin's Sixtieth Birthday, 393-443. MR 2804260

[7] F. Morel and V. Voevodsky, $\mathbf{A}^{1}$-homotopy theory of schemes, Inst. Hautes Études Sci. Publ. Math. 90 (1999), 45-143 (2001). MR 1813224 (2002f:14029)

[8] A. Neeman, The Grothendieck duality theorem via Bousfield's techniques and Brown representability, J. Amer. Math. Soc. 9 (1996), no. 1, 205-236. MR 1308405 (96c:18006)

[9] A. Neeman, Triangulated categories, Annals of Mathematics Studies 148, Princeton University Press, Princeton, NJ, 2001. MR 1812507 (2001k:18010)

[10] I. Panin, K. Pimenov and O. Röndigs, A universality theorem for Voevodsky's algebraic cobordism spectrum, Homology, Homotopy Appl. 10 (2008), no. 2, 211-226. MR 2475610 (2010b:55006)

[11] P. Pelaez, Mixed motives and the slice filtration, C. R. Math. Acad. Sci. Paris 347 (2009), no. 9-10, 541-544. MR 2576905 (2010m:14030)

[12] P. Pelaez, On the functoriality of the slice filtration, preprint (2010).

[13] P. Pelaez, Multiplicative properties of the slice filtration, Astérisque (2011), no. 335 , xvi+289 pp. MR 2807904

[14] D. Quillen, On the formal group laws of unoriented and complex cobordism theory, Bull. Amer. Math. Soc. 75 (1969), 1293-1298. MR 0253350 (40 \#6565)

[15] D. Quillen, Elementary proofs of some results of cobordism theory using Steenrod operations, Advances in Math. 7 (1971), 29-56. MR 0290382 (44 \#7566)

[16] D.G. Quillen, Homotopical algebra, Lecture Notes in Mathematics 43, SpringerVerlag, New York, 1967. MR 0223432 (36 \#6480)

[17] J. Riou, Algebraic $K$-theory, $\mathbf{A}^{1}$-homotopy and Riemann-Roch theorems, $J$. Topol. 3 (2010), no. 2, 229-264. MR 2651359 (2011f:19001) 
[18] O. Röndigs and P.A. Østvær, Modules over motivic cohomology, Adv. Math. 219 (2008), no. 2, 689-727. MR 2435654 (2009m:14026)

[19] M. Spitzweck, Relations between slices and quotients of the algebraic cobordism spectrum, Homology, Homotopy Appl. 12 (2010), no. 2, 335-351. MR 2771593 (2011m:14033)

[20] M. Spitzweck and P.A. Østvær, The Bott inverted infinite projective space is homotopy algebraic K-theory, Bull. Lond. Math. Soc. 41 (2009), no. 2, 281292. MR 2496504 (2010f:19005)

[21] G. Vezzosi, Brown-Peterson spectra in stable $\mathbb{A}^{1}$-homotopy theory, Rend. Sem. Mat. Univ. Padova 106 (2001), 47-64. MR 1876212 (2002j:55007)

[22] V. Voevodsky, Homology of schemes, Selecta Math. (N.S.) 2 (1996), no. 1, 111-153. MR 1403354 (98c:14016)

[23] V. Voevodsky, On the zero slice of the sphere spectrum, Tr. Mat. Inst. Steklova 246 (2004), Algebr. Geom. Metody, Svyazi i Prilozh., 106-115. MR 2101286 (2005k:14042)

[24] V. Voevodsky, $\mathbf{A}^{1}$-homotopy theory, Proceedings of the International Congress of Mathematicians, Vol. I (Berlin, 1998), Doc. Math. 1998, Extra Vol. I, pp. 579-604 (electronic). MR 1648048 (99j:14018)

[25] V. Voevodsky, Open problems in the motivic stable homotopy theory. I, Motives, polylogarithms and Hodge theory, Part I (Irvine, CA, 1998), Int. Press Lect. Ser. 3, Int. Press, Somerville, MA, 2002, pp. 3-34. MR 1977582 (2005e:14030)

Pablo Pelaez pablo.pelaez@uni-due.de

Universität Duisburg-Essen, Mathematik, 45117 Essen, Germany 